\title{
Manipulation of microalgal lipid production: a genetic engineering aspect
}

\begin{abstract}
Interests in microalgal lipids as green and renewable energy sources are piquing as cheap hydrocarbon fossil fuels reach their limit. Lipids from microalgae have important human uses, i.e., energy, food, and pharmaceuticals, depending on its quantity and quality. Genetic engineering is the introduction or suppression of a target gene for the selective expression of a bio-product, e.g., hydrocarbons for fuel or polyunsaturated fatty acids (PUFAs) for food, at a favorable quantity. Past studies like nitrogen starvation or salinity stress have shown to increase lipid contents of microalgae; however, studies on the molecular mechanisms underlying these stress-induced lipid productions remain limited. Next, complementing environmental stress manipulation with genetic engineering would potentially be a better and more effective approach to increase microalgae lipid production and accumulation. There are generally two approaches to enhance microalgae lipid production on a molecular level: firstly, overexpression and improvement of key enzymes involved in fatty acid and isoprenoid biosynthesis and, secondly, repression of lipid catabolic and competitive pathways such as beta-oxidation and starch synthesis. This review provides an update of microalgae lipid research findings to date and aims to address recent system biology discoveries and approaches on microalgae lipid production, the roadblocks encountered, and help needed to realize the ultimate goal, that is, microalgal lipids as sustainable resources for energy and high-value products.
\end{abstract}

Keywords: Microalgae; Lipid content; System biology; Genes manipulation; Genetic engineering 